\title{
Development of Tuberculosis in Child During Treatment with Tumour Necrosis Factor-Alpha Inhibitor Agent: Could This Have Been Prevented?
}

\author{
Iveta Ozere $^{* 1}$, Ruta Šantere ${ }^{2}$ and Anita Skangale ${ }^{3}$ \\ ${ }^{1}$ Riga Stradinšs University, Latvia \\ ${ }^{2}$ Children's Clinical University Hospital, Latvia \\ ${ }^{3}$ Riga East Clinical University Hospital, Centre for Tuberculosis and Lung Diseases, Latvia \\ Received: 棒 June 15, 2018; Published: 彗July 20, 2018 \\ *Corresponding author: Iveta Ozere, Riga Stradinš University, Riga, Latvia, Tel: 37129136790; Email: Iveta.Ozere@rsu.lv
}

\begin{abstract}
Patients treated with anti-TNF- $\alpha$ agents are at increased risk of reactivating latent tuberculosis infection. Screening for tuberculosis infection and disease, and appropriate treatment before commencing of anti-TNF therapy is therefore important. However, currently recommended screening tools (tuberculin skin test, interferon-gamma releasing assays, and chest radiograph) may not detect tuberculosis infection in all children. This may result in development of active tuberculosis during TNF- $\alpha$ inhibitor therapy.
\end{abstract}

Keywords: Anti-TNF- $\alpha$ agents; Tuberculosis; Juvenile Idiopathic Arthritis; Children

Abbreviations: TNF- $\alpha$ : Tumor Necrosis Factor Alpha; TB: Tuberculosis; Mbt: Mycobacterium Tuberculosis; JIA: Juvenile Idiopathic Arthritis; LTBI: Latent Tuberculosis Infection; TST: Tuberculin Skin Test; IGRAs: Interferon-Gamma Releasing Assays; CXR: Chest Radiograph; CT: Computed Tomography

\section{Introduction}

Tumor necrosis factor alpha (TNF- $\alpha$ ) is a pro-inflammatory cytokine that plays a critical role in the host response to tuberculosis (TB), having an essential role in the granuloma formation and maintenance of the latency of TB. Blocking TNF- $\alpha$ in cases of active infection with Mycobacterium tuberculosis (Mbt) results in a failure to form tight granuloma's as well as disintegration of existing granulomas leading to mycobacterial dissemination in a murine model [1-2]. Treatment with anti- TNF- $\alpha$ agents has become an important cornerstone in the treatment of severe, nonremitting forms of juvenile idiopathic arthritis (JIA). However, patients treated with TNF- $\alpha$ inhibitors (in particular, monoclonal antibody preparations, such as infliximab and adalimumab) are at increased risk of reactivating latent tuberculosis infection (LTBI) of approximately two - to eightfold [3-5]. Screening for TB infection and TB disease, and appropriate treatment before commencing of anti-TNF therapy is therefore important [6-7]. Either tuberculin skin test (TST) or interferon-gamma releasing assays (IGRAs) or both, clinical symptom screen for TB disease, and chest radiograph (CXR) are commonly recommended screening tools by most guidelines [8]. Latvia is among five high TB incidence countries in European Union, with a total TB notification rate of 31,3/100 000 in 2015. According to the National Guidelines, all patients are screened for
LTBI (TST or IGRA test or both) and TB disease (symptom screen and CXR) before starting treatment with anti- TNF- $\alpha$ agents. Here we report a case of TB which developed in child during treatment with adalimumab.

\section{Case Report}

A nine-year-old male patient was hospitalized at children's Clinical University Hospital on June 16, 2015 due to complaints of high temperature for two weeks, headache, abdominal distension, loss of weight, pain in the right hip, knee and ankle joints. The child was diagnosed with JIA, oligoarthritis, HLA B-27 negative at the age of 4 years in 2010. In February 2012 at the age of 6 years therapy with methotrexate 7-5mg once per week was started. Therapy was tolerated poorly, JIA progressed with involvement of multiple joints. On October 2014, at the age of 8 years treatment with adalimumab was commenced. Prior treatment screening for LTBI and TB disease was performed: T-SPOT. TB test (Oxford Immunotec, Abingdon, UK) was negative, CXR was normal. Since autumn 2014 the child had a non-troublesome recurrent cough. Consultation of rheumatologist on March 2015 did not reveal any respiratory pathology.

Gradual clinical deterioration started since the end of May 2015, when high temperature, loss of weight, headache, abdominal 
distention, and pain in multiple joints appeared. Abdominal examination in the middle of June revealed ascites, hepatomegaly, splenomegaly, enlarged up to $1-5 \mathrm{~cm}$ in size mesenteric lymph nodes. Computed tomography (CT) of the lungs revealed pleural collection in the right haemithorax, nodular shadows in both lungs, small calcifications in left bronchopulmonary lymph nodes (Figure 1). Blood count analysis demonstrated hypochromic anaemia, blood biochemistry showed increased CRP up to 38mg/L. T-SPOT. TB test was positive on June 26, 2015. Mbt DNS by Xpert ${ }^{\circledR}$ MTB/RIF (Cepheid, Sunnyvale, CA, USA) was positive in low concentration without resistance to Rifampicin from gastric aspirate sample on June 26, 2015. Skeletal scintigraphy revealed arthritis in hip, knee and pedal joints, showing deterioration comparing with previous examination.

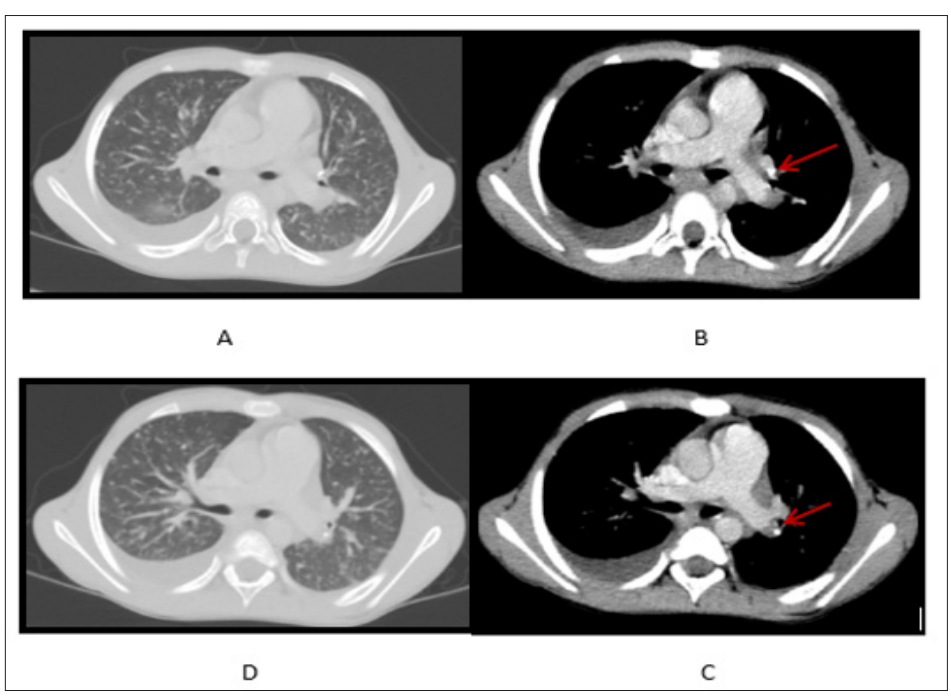

Figuer 1: (A,D) Nodular shadows in lung parenchima and pleural collection in the right haemithorax; (B,C) Calcified bronchopulmonary lymph nodes (arrows).

Because continuous clinical deterioration raised concerns about possible underlying connective tissue disease associated pulmonary damage transthoracic lung biopsy was performed on June 29, 2015. Histopathological investigation revealed tuberculosis granulomata. Finally the child was diagnosed with generalized tuberculosis (disseminated pulmonary TB, TB polyserositis). Standard anti-tuberculosis treatment regimen was started on June 26, 2015 and was continued for 12 months. TB was cured. The child had received BCG vaccination at birth and had post-BCG scar $6 \mathrm{~mm}$. No particular contact with TB patients was identified.

\section{Discussion}

Our patient developed severe TB during 8 months of the treatment with adalimumab, despite the fact that LTBI and TB disease were excluded before therapy. Taking into account the densely calcified lymph nodes identified on CT, one can speculate, that TB infection was old, acquired in the past, and not diagnosed by conventional screening methods prior treatment with adalimumab. It has been shown that CXR can have low-sensitivity and specificity in detecting TB abnormalities. Chest CT is superior to CXR in the visualization of intrathoracic lymphadenopathy, mild parenchymal abnormality and early cavitation [9]. CT scans in addition to CXR revealed calcification in lymph nodes and lung parenchyma in $42 \%$ of children and adolescents diagnosed with TB in study by Ziemele et al [10]. Thought old calcified TB lesions are uniformly negative for culture and acid fast- staining, such lesions are frequently found in lungs in reactivation TB indicating their association with reactivation. In the study by Iida et al. [11], it was shown that old TB foci, particularly those exhibiting some calcification, all were negative for culture and acid-fast staining, however contained abundant Mbt components. All of the calcified granulomatous lesions examined were positive by real-time PCR and contained granules with mycobacterial cell wall components, some of which had bacillus shape. Authors explained low sensitivity of microscopy and culture by low proliferation rate of Mbt and loss of acidfastness in environmental stress conditions, and recognized, that the risk of reactivation of latent TB from calcified lesions should be considered, especially in patients who are immunocompromised.

T-SPOT. TB test detects cell-mediated IFN- $\gamma$ responses to Mbt-specific antigens. Because the amount of Mbt is small in LTBI patients, diagnosis of this condition mainly depends on the immune response of the host rather than the bacteria itself Use of Methotrexate and chronic inflammatory disease itself could possibly reduce the sensitivity of T-SPOT.TB test during latency of TB infection in our patient [12].

\section{Conclusion}

The sensitivity of currently recommended screening tools for LTBI and TB disease was insufficient to reveal calcified lymph nodes which were likely the hotbeds of reactivation of Mbt infection. In children living in high $\mathrm{TB}$ incidence settings who receive immunosuppressive drugs and who are candidates for treatment with anti- TNF- $\alpha$ agents, consideration should be given for lung CT investigation after careful risk-benefit ratio consideration. 


\section{References}

1. Calzada-Hernández J, Anton-López J, Bou-Torrent R, Iglesias-Jiménez E, Ricart-Campos S, et al. (2015) Tuberculosis in pediatric patients treated with anti-TNF $\alpha$ drugs: a cohort study. Pediatric Rheumatology 13: 54.

2. Dekker L, Armbrust W, Rademaker CMA, Prakken B, Kuis W, et al. (2004) Safety of anti-TNF therapy in children with juvenile idiopathic Arthritis. Clin Exp Rheumatol 22: 252-258.

3. Davies HD, Aap Commitee On Infectious Diseases (2016) Infectious Complications with the Use of Biologic Response Modifiers in Infants and Children. Pediatrics 138(2): e20161209.

4. Esmail H, Wilkinson RJ (2017) Minimizing Tuberculosis Risk in Patients Receiving Anti-TNF Therapy. Ann Am Thorac Soc 14(5): 621-623.

5. Viladrich Mir I, Dauden Tello DE, Solano-Lopez G, Lopez Longo FJ, Samso Taxonera Samso C, et al. (2016) Documento de consenso sobre la prevencion y el tratamiento de la tuberculosis enpacientes candidatos a tratamiento biologico. Arch Bronconeumol 52: 36-45.

6. Solovic I, Sester M, Gomez Reino JJ, Rieder HL, Ehlers S, et al. (2010) The Risk of Tuberculosis Related to Tumour Necrosis Factor Antagonist
Therapies: a TBNET consensus statement. European Respiratory Journal 36(6): 1185-1206.

7. WHO (2015) Guidelines on the management of latent tuberculosis.

8. Hewitt RJ, Francis M, Singanayagam A, Kon OM (2015) Screening tests for tuberculosis before starting biological therapy. BMJ 350: h1060.

9. Garrido J B, Alias Hernandez I, Bonillo Perales A, Rubí Ruiz T, González Jiménez Y, et al. (2012) Usefulness of thoracic CT to diagnose tuberculosis disease in patients younger than 4 years of age. Pediatr Pulm 47: 895902.

10. Ziemele B, Ranka R, Ozere I (2017) Pediatric and adolescent tuberculosis in Latvia, 2011-2014: case detection, diagnosis and treatment Int J Tuberc Lung Dis 21(6): 637-645.

11. Iida T, Uchida K, Lokman N, Furukawa A, Suzuki Y, et al. (2014) Calcified Granulomatous Lung Lesions Contain Abundant Mycobacterium Tuberculosis Components. J Mycobac Dis 4(1): 142.

12. Jing-Wen A, Qiao-Ling R, Qi-Hui L, Wen-Hong Z (2016) Updates on the risk factors for latent tuberculosis reactivation and their managements. Emerging Microbes and Infections 5(2): e10.

\section{ISSN: 2574-1241}

DOI: $10.26717 / B J S T R .2018 .07 .001452$

Iveta Ozere. Biomed J Sci \& Tech Res

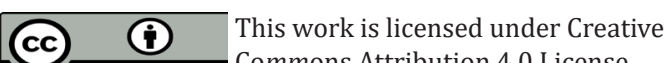

Submission Link: https://biomedres.us/submit-manuscript.php
This work is licensed under Creative Commons Attribution 4.0 License

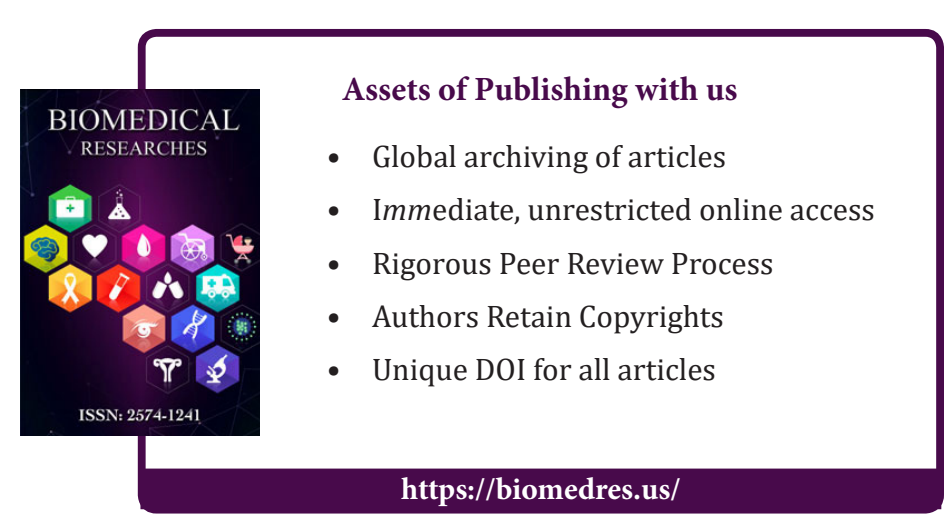

